Reporte de casos

\title{
Retención de caninos permanentes: reporte de un caso y revisión de literatura
}

\author{
Mario Alberto-Maldonado- Ramírez ${ }^{1}$ \\ Hilda-Hernández-Isassi ${ }^{2}$ \\ Dolores-Castillo- Leyva ${ }^{2}$ \\ Jorge Alberto-Quezada-Castillo ${ }^{2}$ \\ Dolores-Lazcano-Velázquez²
}

\section{Resumen}

Pacientes con caninos permanentes retenidos, frecuentemente sufren de extracciones, presen- tamos un caso con cuatro caninos permanentes retenidos y la revisión de la literatura.

Palabras clave: diente retenido,exodoncia

Relato de caso

\section{Retenção de caninos permanentes: relato de caso clínico e revisão da literatura}

Resumo

Pacientes com caninos permanentes retidos, frequentemente sofrem de extraçoes dentarias, apresentamos um caso com quatro caninos permanentes retidos e a revisão da literatura.

Palavras-chave: extração, dente impactado,

Case report

\section{Permanent canines retention: a case report and literature review}

\begin{abstract}
Patients with retained permanent canines often suffer from extraction; we present a case with

four permanent canines retained and literature review.

Keywords: impacted tooth, extraction. 


\section{Introducción}

La retención de caninos maxilares ha sido descrita con anterioridad, la causa aparente de esto suele ser la falta de espacio y / o pérdida de guía canina para la erupción, es el segundo diente con mayor frecuencia de impactación, después de los terceros molares permanentes.(1)

\section{Epidemiología}

La prevalencia de la retención del canino maxilar varía del $0.9 \%$ al $2 \%$, pero se pueden encontrar reportes con hasta un 7\%(2), tiene predilección por el género femenino, así como por la posición palatina con respecto a la labial que va de 3:1 hasta 6:1(3) y por lo general se presenta de forma unilateral (4), en proporción de 5:1 y en el lado izquierdo más que en el derecho.(5) En cambio los caninos inferiores se presentan en una proporción 20 veces menor que los superiores.(6) En México existen datos que sugieren que esta anomalía tiene una prevalencia de $5.8 \%$ en la población(7).

\section{Desarrollo del canino superior}

El desarrollo del canino superior inicia a nivel de la pared anterior del seno maxilar, por debajo del piso de la órbita y desde allí desciende mesial y lingualmente alcanzando una posición vertical una vez que llega al plano oclusal. Su erupción es guiada por la raíz del incisivo lateral permanente, por lo que las alteraciones en este diente, además de otros factores pueden producir cambios en su proceso eruptivo normal. $(1,9,10)$ Se le considera el diente más importante para la continuidad del arco maxilar, ya que provee de un buen contorno a la cara, y estética a la arcada dental.(11) Por el contrario el canino mandibular permanente se forma más cerca del borde inferior de la mandíbula, es la pieza más grande del maxilar inferior por delante de los molares. El vértice de la cúspide de su corona está localizado lingual al ápice de su predecesor. Sin embargo la cara bucal de su corona produce un abultamiento hacia afuera y se puede palpar fácilmente. En la mayoría de los casos el canino inferior emerge primero que el premolar, el cual inicialmente está más cercano al plano oclusal (12), esto permite que no se retenga tan frecuentemente como el canino maxilar.

\section{Secuelas de la retención del canino}

Las secuelas de esta anomalía de la erupción, van desde la simple ausencia clínica con pérdida del contorno maxilar, hasta la reabsorción del incisivo lateral, pasando por la desviación de la línea media, mesialización del sector posterior con pérdida de espacio del lado afectado, transmigración dentaria, giroversión e inclinación de incisivo lateral del lado afectado y en caso de no erupcionar un porcentaje de ellos se asocia a la formación de quistes con sus posibles complicaciones; según Howell, el 16\% de los seres humanos presentan algún tipo de diente retenido.(12) Otras estadísticas nos demuestran que el 30\% de los dientes retenidos presentan formación de quistes dentigeros, que el 35\% de los Ameloblastomas están asociados a quistes Dentígeros y, que el 51\% de los Queratoquistes también lo están. Aunque el desarrollo de un Ameloblastoma a partir de un quiste dentigero, es la complicación más corriente, la capacidad que tienen las células epiteliales del revestimiento quístico de transformarse en "secretoras" $y$, en epitelio "escamoso estratificado" constituyen la base para el desarrollo de un carcinoma mucoepidermoide intraoseo o un carcinoma de células escamosas en el ángulo de la mandíbula, $(14,15,16)$ esto convierte a los caninos (y molares) retenidos en 
piezas que se pueden tornar peligrosas por su alto riesgo a la transformación patológica.

Por ello, un diagnóstico temprano y un seguimiento cuidadoso del desarrollo de la dentición son esenciales para la intervención temprana y la corrección de las anomalías eruptivas.(1)

\section{Manejo clínico}

El manejo clínico se puede clasificar en cuatro vertientes prácticamente:

1. Extracción quirúrgica del, o los caninos retenidos y posterior cierre de espacios.

2. Extracción de premolares, abordaje quirúrgico de canino(s) retenido(s), tracción y posicionamiento en el arco.

3. Distalización de sector posterior para la obtención de espacio(s), abordaje quirúrgico, tracción y posicionamiento en el arco y

4. Expansión de maxilar(es) por medios ortopédicos para la creación del(os) espacio(os) faltante(es) para el o los caninos retenido(s) en combinación con alguno(s) de los anteriores.

Sin duda su manejo representa todo un reto para el odontólogo que lo enfrenta, ya que sue-

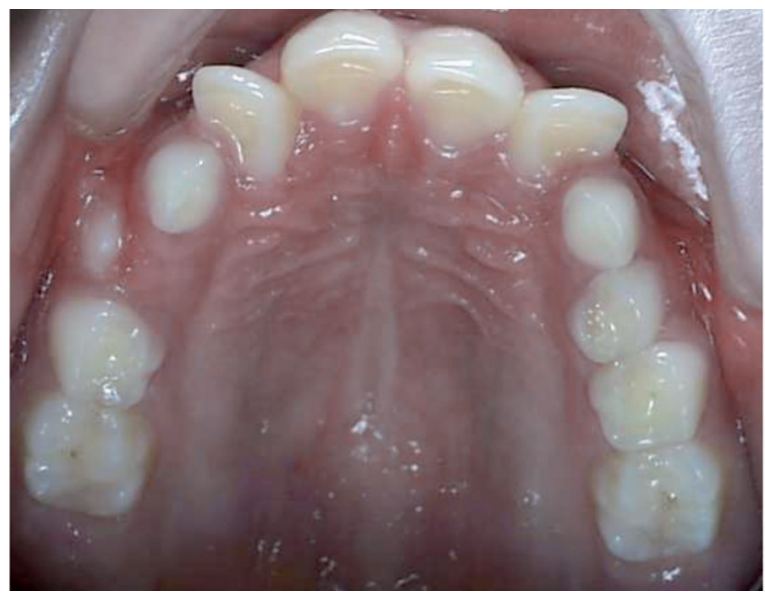

Fig. 5. Vista Intraoral Superior Inicial.

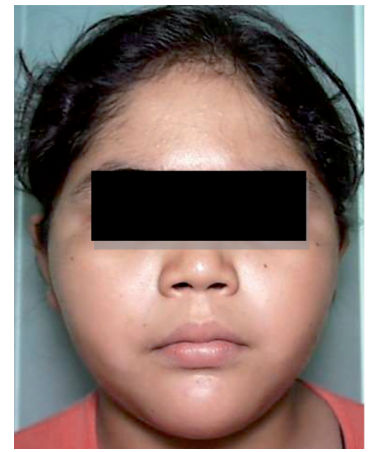

Fig. 1. Vista frontal.

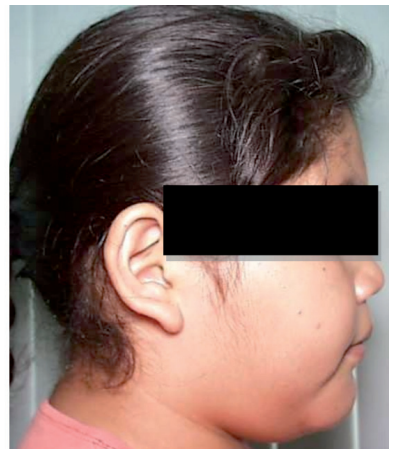

Fig. 2. Vista lateral.

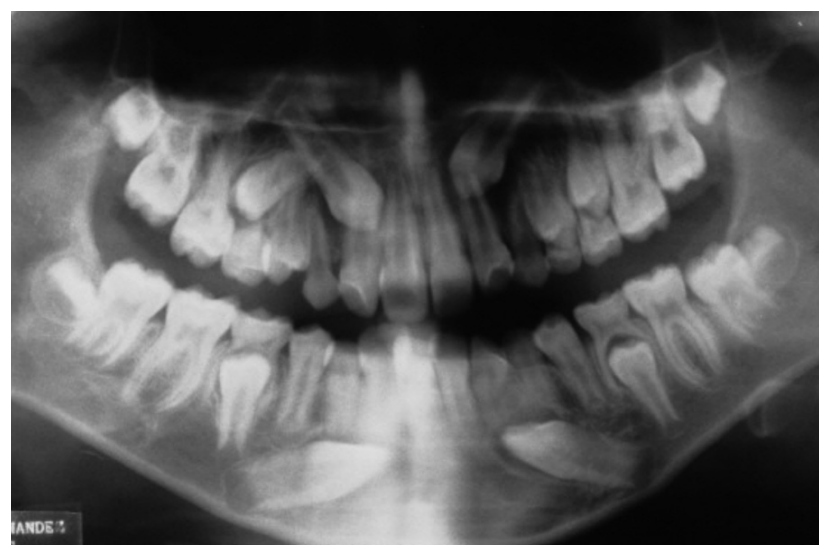

Fig. 3. Radiografía Panorámica Inicial.

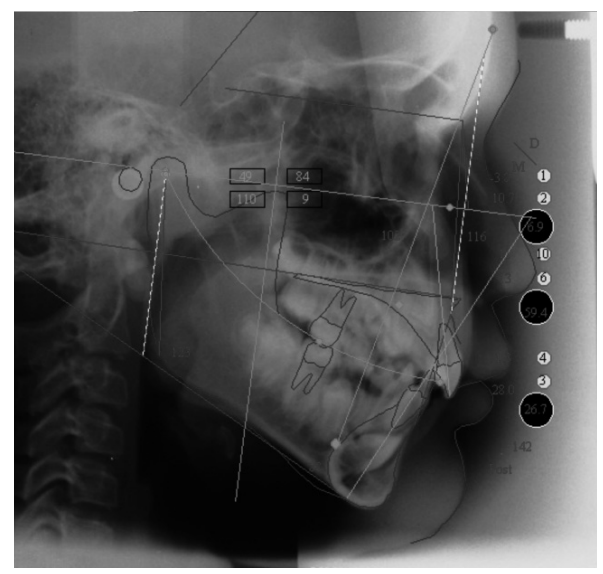

Fig. 4. Radiografía Lateral.

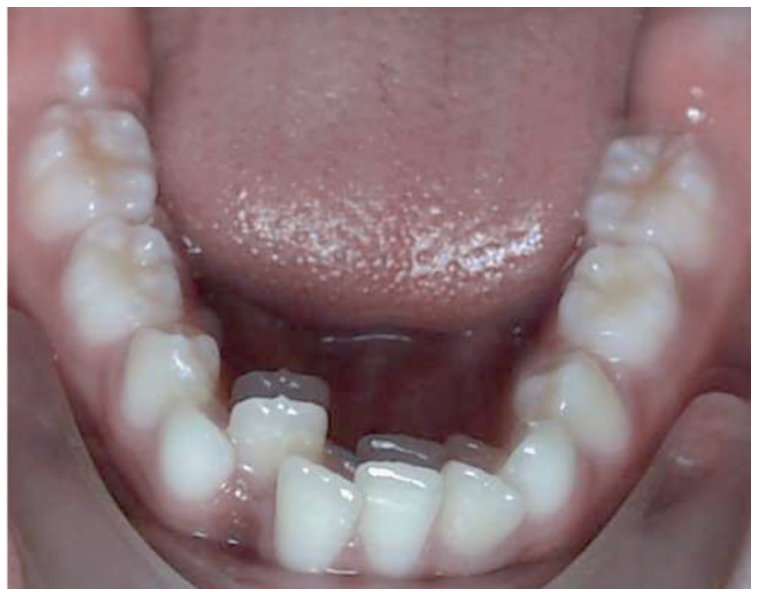

Fig. 6. Vista Intraoral Inferior Inicial. 
len estar presentes otras alteraciones en los arcos maxilares.

Presentamos el siguiente caso clínico utilizando la clasificación propuesta por Ugalde F. (17)

\section{Caso clínico completo}

Paciente femenino de 10 años de edad que acude a consulta por primera vez solicitando tratamiento de ortodoncia, el examen clínico mostro: malposición dentaria y discrepancia intermaxilar, por lo que se solicitaron estudios complementarios, radiografía Panorámica y Lateral de Cráneo, Modelos de Estudio y Fotografías.

\section{Diagnóstico anteroposterior}

- Clase esqueletal: clase II.

- Relación molar: clase II, subdivisión I.

- Perfil: convexo.

- Curva de Spee: profunda.

- Incisivos superiores retroinclinados (centrales).

- Incisivos inferiores retroinclinados.

- Overjet: $8 \mathrm{~mm}$.

\section{Diagnóstico vertical}

- Tipo de crecimiento: Dolicocefálico.

- Sobremordida vertical: $4 \mathrm{~mm}$.

\section{Diagnóstico transversal}

- Apiñamiento: arcada superior $4.5 \mathrm{~mm}$, arcada inferior $7.5 \mathrm{~mm}$.

- Mordida cruzada: no.

- Línea media: Inferior desviada a la derecha.
- Forma de las arcadas: Superior: ovoide Inferior: triángular.

- No existen asimetrías faciales, ni laterognasias.

Diagnóstico definitivo: Maloclusión clase II con retención bilateral de caninos maxilares y mandibulares.

\section{Formato para el diagnóstico de caninos retenidos}

\begin{tabular}{|c|c|c|}
\hline Ubicación & Maxilar & Mandibular \\
\hline Ubicación & Bilateral & Bilateral \\
\hline Profundidad & Moderado & Profundo \\
\hline Angulación & Oblicua & Horizontal \\
\hline Presentación & Central & Central \\
\hline Edo. Radicular & Formación & En formación \\
\hline Daño a adyacentes & Ninguno & Ninguno \\
\hline
\end{tabular}

\section{Etiología}

Discrepancia de longitud de arco debido a: Apiñamiento anterior y Mesialización del sector posterior.

\section{Plan de tratamiento}

\begin{tabular}{|c|c|}
\hline Arcada superior & Arcada inferior \\
\hline $\begin{array}{c}\text { Exodoncia de 53, 54, 55, } \\
63,64 \text { y } 65\end{array}$ & $\begin{array}{c}\text { Exodoncia de 73, 75, 83 } \\
\text { y } 85\end{array}$ \\
\hline $\begin{array}{c}\text { Cirugía para descubrir: } \\
13 \text { y } 23\end{array}$ & $\begin{array}{c}\text { Cirugía para descubrir: } \\
33 \text { y } 43\end{array}$ \\
\hline $\begin{array}{c}\text { Aliviar apiñamiento } \\
\text { (expansión) }\end{array}$ & $\begin{array}{c}\text { Aliviar apiñamiento } \\
\text { (expansión) }\end{array}$ \\
\hline $\begin{array}{c}\text { Abrir espacio para } \\
\text { diente no. } 23\end{array}$ & $\begin{array}{c}\text { Abrir espacio para } 33 \\
\text { y } 43\end{array}$ \\
\hline Tracción de: 13 y 23 & Tracción de 33 y 43 \\
\hline Alinear y nivelar & Alinear y nivelar \\
\hline Ligas finales & Ligas finales \\
\hline Retención & Retención \\
\hline
\end{tabular}


- $\quad$ El $2^{\circ}$ Premolar superior derecho (sin emerger) después de llevar a cabo una evaluación se decidió no realizar cirugía alguna para su exposición y se vigiló su erupción ya que el desarrollo radicular se encontraba en fase inicial y las posibilidades de corregir su dirección hacía una zona de menor resistencia habiendo realizado la exodoncia de 55, eran mayores, como lo demuestra la secuencia radiográfica.

\section{Programa de trabajo}

Previo acuerdo con el cirujano maxilofacial y después de realizar la exodoncia de dientes primarios, se programo la cirugía para exponer los caninos y poder adherirles un bracket y colocarles una ligadura elástica en cadena, se cemento aparatología fija de expansión: Arco W en maxilar y Arco lingual activo en mandíbula, se realizo tracción muy ligera colocando el extremo libre de la ligadura en cadena en el extremo libre del arco W de cada lado, la activación se llevó a cabo pasando un eslabón de la cadena cada 3 semanas, esto mantuvo la cadena elástica lo suficientemente activa sin ejercer una fuerza de tracción excesiva, esta fuerza es suficientemente para traccionar al diente manteniendo integro el paquete neurovascular ya que su ruptura está asociada a necrosis postratamiento, una vez obtenido el espacio y corregido parcialmente la inclinación de los caninos (aún sin emerger) se co-

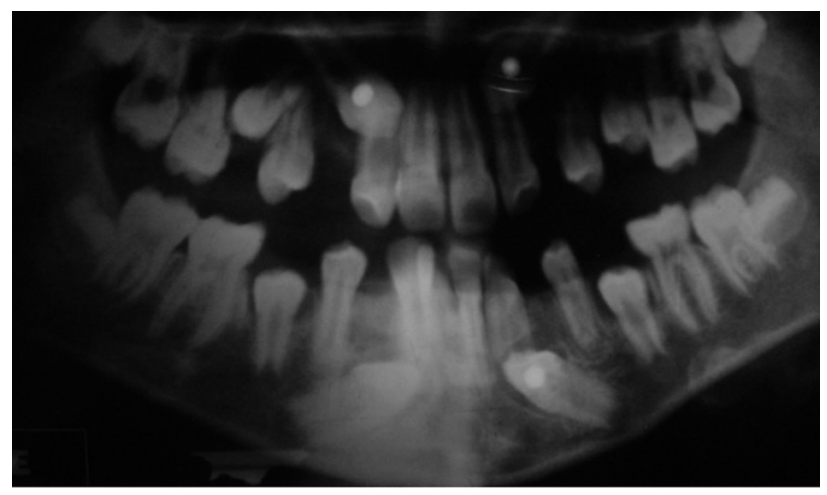

Fig. 7. Rx Panorámica pos extracciones. locaron brackets (Roth pre-ajustados slot 18) en centrales y premolares de ambas arcadas dejando libres a los laterales teniendo cuidado para no colocar las raíces en el camino de los caninos, se retiraron arco $\mathrm{W}$ y Lingual, la secuencia de arcos se inició con arcos NiTi redondos .10, .12, .14 , cuadrado .16x.16, rectangular .16x.18 y rectangular acero .17x.22 y .18x.25, los premolares fueron fijados con ligadura de alambre para evitar pérdida de anclaje ya que la cadena se fijo en la extensión del bracket para continuar enderezando los caninos, una vez puestos en posición vertical y clínicamente visibles, se colocaron brackets en laterales y se continuo el tratamiento hasta colocar en posición los cuatro caninos.

\section{Pronóstico}

El pronóstico fue reservado ya que la posición central represento una cirugía difícil y compleja, la profundidad de los caninos requirió de múltiples activaciones para poder llevarlo al arco sin provocar fenestración ni reabsorción radicular de incisivos laterales ni del canino mismo, ni ruptura del paquete neurovascular para mantener la vitalidad pulpar de los caninos, además dada la complejidad del caso, el tiempo de tratamiento sería mayor.

\section{Conclusiones}

La retención de caninos maxilares y/o mandibulares representa uno de los retos más difíciles

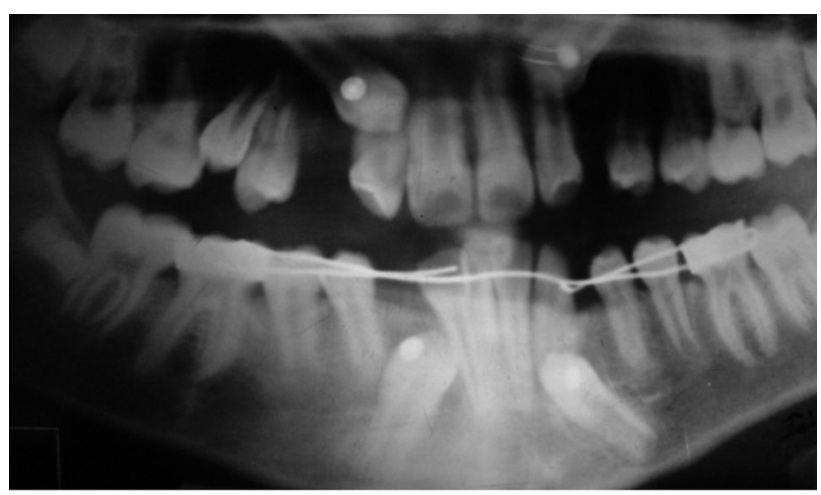

Fig. 8. Rx 6 meses de inicio de dientes primarios. 


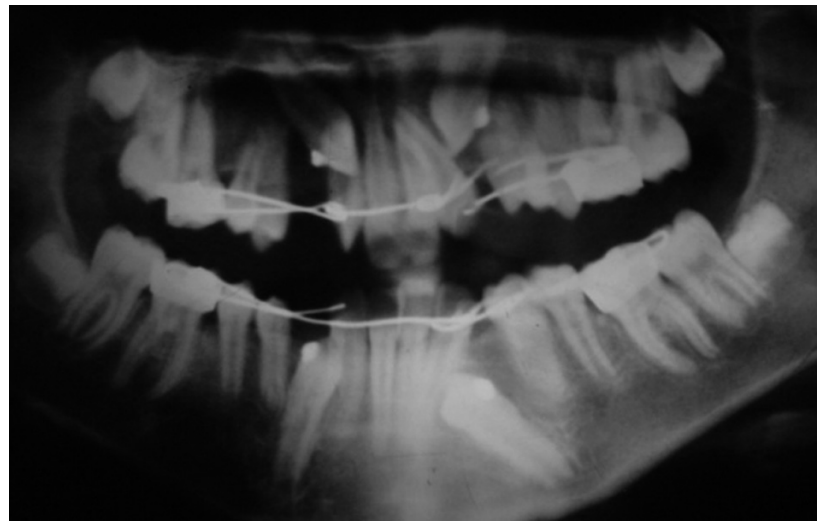

Fig. 9. Rx 9 meses de inicio.

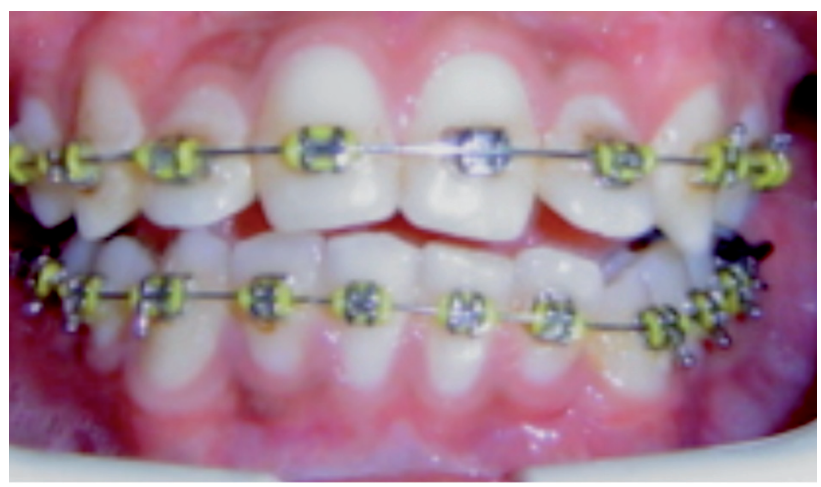

Fig. 11. Vista a los 18 meses.

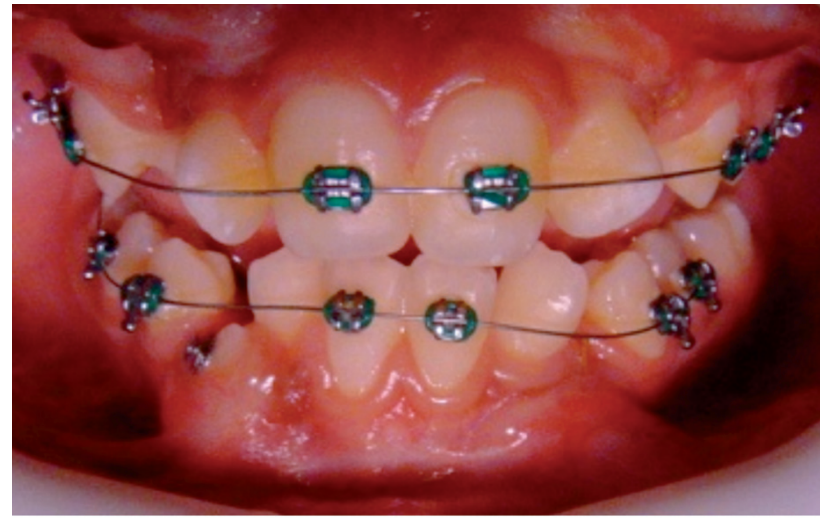

Fig. 10. Brackets a los 12 meses de inicio.

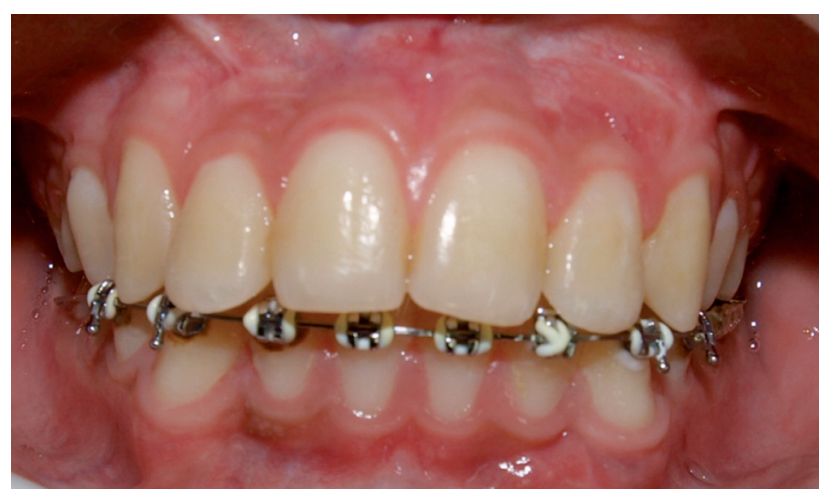

Fig. 12. Vista a los 24 meses.

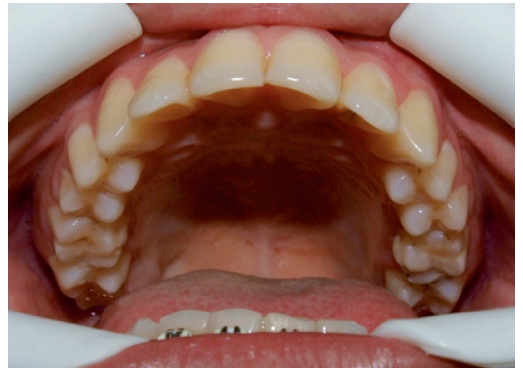

Fig. 13. Arcada superior.

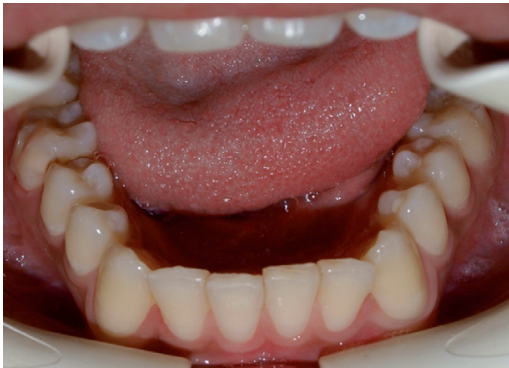

Fig. 14. Arcada inferior.

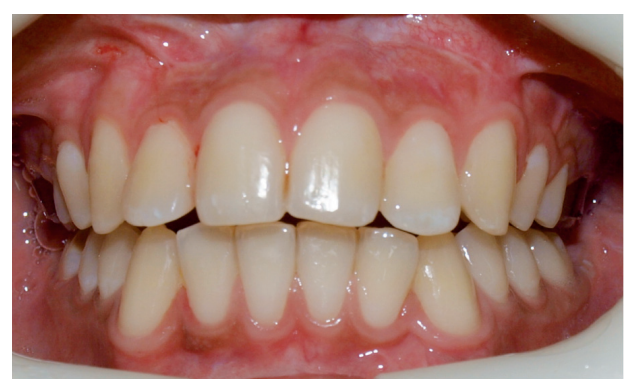

Fig. 15. Control 8 años post tratamiento. de resolver para el especialista, ya que no sólo debe tomar la decisión de extraer o no extraer, sino que debe seleccionar la pieza dental más adecuada si se decide por realizar extracciones, además el tratamiento suele ser complejo si de- cide no extraer piezas, sin duda son estos casos en donde se resalta la importancia de un buen diagnóstico y plan de tratamiento ya que representan los pilares para una buena toma de decisiones. 


\section{Referencias}

1. Pérez F. M., Pérez F. P., Fierro M. C. Alteración en la Erupción de Caninos Permanentes. Int. J. Morphol, 2009; 27(1): 139-143.

2. Ericson, $\mathrm{S}$ and Kurol, J. Early treatment of palatally erupting maxillary canines by extraction of the primary canines. Eur J. Orthod. 1998; 10: 283

3. Rimes R, Mitchell C, Willmot D. Maxillary incisor root resorption in relation to the canine: a review of 26 patients. Eur. J. Orthod. 1997; 19(1): 79-84.

4. Ugalde M. F. y González L. R. Prevalencia de retenciones de caninos en pacientes tratados en la clínica de ortodoncia de UNITEC. ADM, 1999; Vol. LVI, N², Marzo-Abril:49-58.

5. Kuftinee M, Storn D and Shapira Y. The impacted maxillary canine. Part I Review of concepts and Part II Clinical approarches and solutions. J. Dent. Children. 1995; 63 (5): 325-334.

6. http://www.hospitalgea.salud.gob.mx/descargas/Art_Can_Inf_Reten.pdf

7. Vera M. C., Mourelle M. M., Nova G. J. El canino superior incluido. Orientaciones diagnósticas y terapéuticas para el odontólogo general. Profesión Dental. 2000; 3(6): 356-361.

8. Zivov L.A. / Solé V.P. CIRUGIA BUCAL PARA PREGRADO Y EL ODONTÓLOGO GENERAL. Cap. 8, Pag. 341 Disponible en: http: / / media.axon.es/pdf/88068_2.pdf

9. Hung-Huey T. Eruption process of upper permanent canine. The Journal of Clinic Pediatric Dentistry. 2001; 24(3).

10. Peñarrocha M. A, Peñarrocha M, Larrazábal C, García B. Caninos incluidos, consideraciones quirúrgicas y ortodóncicas. Arch. Odontoestomatol. 2003; 19(5): 336-46.

11. Johnston W. Treatment of palatally canine teeth. Am.J.Ortho. 1969; 56 (6): 589-596.

12. López J. J. Tratamiento quirúrgico-ortodóncico de los caninos incluidos. Revisión de la literatura y aportación de casos clínicos. Revista Europea de Odonto-Estomatologia. 2001; XIII (2).

13. Kruger O. Trat. Cirugía Bucal 4ta Edición, 1976,Editorial Interamericana.

14. Cabrini R. L. Anatomía Patológica Bucal. 1988; Cap. Quistes de los Maxilares. Edit. Mundi.

15. Peña C.A. y Scat N.J. Quiste Dentígero ¿Patología de origen iatrogénico? Disponible en: www.odonto32.com

16. Sapp P.J., Eversole L.R. y Wysocki G.P. Patología Oral y Maxilofacial Contemporánea. 2a . Edición, 2004; Edit. Elsevier Mosby.

17. Ugalde M.F. Clasificación de caninos retenidos y su aplicación clínica. ADM. 2001; Vol. LVII, N 1, En-Feb:21-30.

Recibido 07-08-12

Aceptado: 01-12-12

Correspondencia: odo.pediatrica.mario@gmail.com 